\title{
Higher-order identities for balancing numbers
}

\author{
Takao Komatsu \\ Department of Mathematics, School of Science \\ Zhejiang Sci-Tech University \\ Hangzhou 310018 China \\ e-mail: komatsuezstu.edu.cn
}

Received: 1 November 2019

Revised: 15 June 2020

Accepted: 16 June 2020

\begin{abstract}
Let $B_{n}$ be the $n$-th balancing number. In this paper, we give some explicit expressions of $\sum_{l=0}^{2 r-3}(-1)^{l}\left(\begin{array}{c}2 r-3 \\ l\end{array}\right) \sum_{\substack{j_{1}+\cdots+j_{r}=n-2 l \\ j_{1}, \ldots, j_{r} \geq 1}} B_{j_{1}} \cdots B_{j_{r}}$ and $\sum_{\substack{j_{1}+\cdots+j_{r}=n \\ j_{1}, \ldots, j_{r} \geq 1}} B_{j_{1}} \cdots B_{j_{r}}$. We also consider

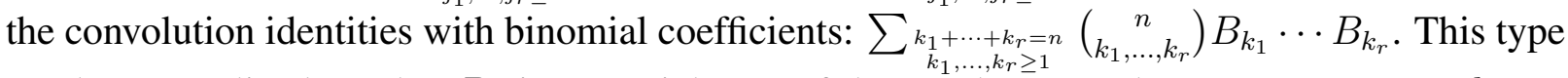
can be generalized, so that $B_{n}$ is a special case of the number $u_{n}$, where $u_{n}=a u_{n-1}+b u_{n-2}$ ( $n \geq 2$ ) with $u_{0}=0$ and $u_{1}=1$.
\end{abstract}

Keywords: Convolutions, Balancing numbers.

2010 Mathematics Subject Classification: Primary 11B39; Secondary 11B83, 05A15, 05A19.

\section{Introduction}

Higher-order convolutions for various types of numbers (or polynomials) have been studied, with or without binomial (or multinomial) coefficients, including Bernoulli, Euler, Genocchi, Cauchy, Stirling, and Fibonacci numbers $[1-3,6-8,10]$. One typical one is due to Euler, given by

$$
\sum_{k=0}^{n}\left(\begin{array}{l}
n \\
k
\end{array}\right) \mathcal{B}_{k} \mathcal{B}_{n-k}=-n \mathcal{B}_{n-1}-(n-1) \mathcal{B}_{n} \quad(n \geq 0)
$$

where $\mathcal{B}_{n}$ are Bernoulli numbers, defined by

$$
\frac{t}{e^{t}-1}=\sum_{n=0}^{\infty} \mathcal{B}_{n} \frac{t^{n}}{n !} \quad(|t|<2 \pi) .
$$

A positive integer $x$ is called balancing number if

$$
1+2+\cdots+(x-1)=(x+1)+\cdots+(y-1)
$$


holds for some integer $y \geq x+2$. The problem of determining all balancing numbers leads to a Pell equation, whose solutions in $x$ can be described by the recurrence $B_{n}=6 B_{n-1}-B_{n-2}$ $(n \geq 2)$ with $B_{0}=0$ and $B_{1}=1$ (see $[4,5]$ ). One of the most general extensions of balancing numbers is when (1) is being replaced by

$$
1^{k}+2^{k}+\cdots+(x-1)^{k}=(x+1)^{l}+\cdots+(y-1)^{l},
$$

where the exponents $k$ and $l$ are given positive integers. In the work of Liptai et al. [12] effective and non-effective finiteness theorems on (2) are proved. In [9] a balancing problem of ordinary binomial coefficients is studied. Using the method of linear forms in logarithms, 1 is the only Fibonacci number [11]. Some more results can be seen in [13,14].

The generating function $f(x)$ of balancing numbers $B_{n}$ is given by

$$
f(x):=\frac{x}{1-6 x+x^{2}}=\sum_{n=0}^{\infty} B_{n} x^{n} .
$$

Then $f(x)$ satisfies the relation:

$$
f(x)^{2}=\frac{x^{2}}{1-x^{2}} f^{\prime}(x)
$$

or

$$
\left(1-x^{2}\right) f(x)^{2}=x^{2} f^{\prime}(x)
$$

The left-hand side of (4) is

$$
\begin{aligned}
& \left(1-x^{2}\right)\left(\sum_{u=0}^{\infty} B_{u} x^{u}\right)\left(\sum_{v=0}^{\infty} B_{v} x^{v}\right) \\
& =\sum_{n=0}^{\infty} \sum_{j=0}^{n} B_{j} B_{n-j} x^{n}-\sum_{n=2}^{\infty} \sum_{j=0}^{n-2} B_{j} B_{n-j-2} x^{n} .
\end{aligned}
$$

The right-hand side of (4) is

$$
x^{2} \sum_{n=1}^{\infty} n B_{n} x^{n-1}=\sum_{n=0}^{\infty}(n-1) B_{n-1} x^{n} .
$$

Comparing the coefficients of both sides, we get

$$
\begin{aligned}
(n-1) B_{n-1} & =\sum_{j=0}^{n} B_{j} B_{n-j}-\sum_{j=0}^{n-2} B_{j} B_{n-j-2} \\
& =\sum_{j=1}^{n-1}\left(B_{j} B_{n-j}-B_{j-1} B_{n-j-1}\right) .
\end{aligned}
$$

Here, notice that $B_{0}=0$. By changing $n$ by $n+1$, we get the following.

Theorem 1. For $n \geq 1$, we have

$$
n B_{n}=\sum_{j=1}^{n}\left(B_{j} B_{n-j+1}-B_{j-1} B_{n-j}\right) .
$$


Differentiating both sides of (3) by $x$ and dividing them by 2, we obtain

$$
f(x) f^{\prime}(x)=\frac{x}{\left(1-x^{2}\right)^{2}} f^{\prime}(x)+\frac{x^{2}}{2\left(1-x^{2}\right)} f^{\prime \prime}(x) .
$$

By (3) and (5), we get

$$
\begin{aligned}
f(x)^{3} & =\frac{x^{2}}{1-x^{2}} f(x) f^{\prime}(x) \\
& =\frac{x^{3}}{\left(1-x^{2}\right)^{3}} f^{\prime}(x)+\frac{x^{4}}{2\left(1-x^{2}\right)^{2}} f^{\prime \prime}(x)
\end{aligned}
$$

or

$$
\left(1-x^{2}\right)^{3} f(x)^{3}=x^{3} f^{\prime}(x)+\frac{1}{2} x^{4}\left(1-x^{2}\right) f^{\prime \prime}(x) .
$$

The left-hand side of (7) is equal to equal to

$$
\begin{aligned}
& \left(1-3 x^{2}+3 x^{4}-x^{6}\right) \sum_{n=0}^{\infty} \sum_{\substack{j_{1}+j_{2}+J_{3}=n \\
j_{1}, j_{2}, j_{3} \geq 0}} B_{j_{1}} B_{j_{2}} B_{j_{3}} x^{n} \\
& =\sum_{l=0}^{3} \sum_{n=2 l}^{\infty}(-1)^{l}\left(\begin{array}{l}
3 \\
l
\end{array}\right) \sum_{\substack{j_{1}+j_{2}+J_{3}=n-2 l \\
j_{1}, j_{2}, j_{3} \geq 1}} B_{j_{1}} B_{j_{2}} B_{j_{3}} x^{n} .
\end{aligned}
$$

The right-hand side of (7) is

$$
\begin{aligned}
& x^{3} \sum_{n=1}^{\infty} n B_{n} x^{n-1}+\frac{x^{4}}{2} \sum_{n=2}^{\infty} n(n-1) B_{n} x^{n-2}-\frac{x^{6}}{2} \sum_{n=2}^{\infty} n(n-1) B_{n} x^{n-2} \\
& =\sum_{n=2}^{\infty} \frac{(n-1)(n-2)}{2} B_{n-2} x^{n}-\sum_{n=4}^{\infty} \frac{(n-4)(n-5)}{2} B_{n-4} x^{n} .
\end{aligned}
$$

Comparing the coefficients of both sides, we get the following result.

Theorem 2. For $n \geq 4$, we have

$$
\sum_{l=0}^{3}(-1)^{l}\left(\begin{array}{l}
3 \\
l
\end{array}\right) \sum_{\substack{j_{1}+j_{2}+J_{3}=n-2 l \\
j_{1}, j_{2}, j_{3} \geq 1}} B_{j_{1}} B_{j_{2}} B_{j_{3}}=\left(\begin{array}{c}
n-1 \\
2
\end{array}\right) B_{n-2}-\left(\begin{array}{c}
n-4 \\
2
\end{array}\right) B_{n-4} .
$$

In this paper, we give some explicit expression of a more general case

$$
\sum_{l=0}^{2 r-3}(-1)^{l}\left(\begin{array}{c}
2 r-3 \\
l
\end{array}\right) \sum_{\substack{j_{1}+\ldots+j_{r}=n-2 l \\
j_{1}, \ldots, j_{r} \geq 1}} B_{j_{1}} \cdots B_{j_{r}}
$$

and

$$
\sum_{\substack{j_{1}+\cdots+j_{r}=n \\ j_{1}, \ldots, j_{r} \geq 1}} B_{j_{1}} \cdots B_{j_{r}}
$$


We also consider a different type for more general numbers:

$$
\sum_{\substack{k_{1}+\cdots+k_{r}=n \\
k_{1}, \ldots, k_{r} \geq 1}}\left(\begin{array}{c}
n \\
k_{1}, \ldots, k_{r}
\end{array}\right) u_{k_{1}} \cdots u_{k_{r}}
$$

where $u_{n}=a u_{n-1}+b u_{n-2}(n \geq 2)$ with $u_{0}=0$ and $u_{1}=1$. When $a=6$ and $b=-1$, this is reduced to the convolution identity for balancing numbers. When $a=b=1$, this is reduced to the convolution identity for Fibonacci numbers. The corresponding identities for Lucas-balancing numbers are also given.

\section{Main results}

First, as a general case of (3) and (6), we can have the following.

Lemma 1. For $r \geq 2$, we have

$$
f(x)^{r}=\frac{x^{2 r-2} f^{(r-1)}(x)}{(r-1) !\left(1-x^{2}\right)^{r-1}}+\sum_{k=1}^{r-2} \frac{\sum_{j=0}^{k-1}\left(\begin{array}{c}
k \\
j
\end{array}\right)\left(\begin{array}{c}
r-2 \\
k-j-1
\end{array}\right) x^{2 r-k+2 j-2}}{k(r-k-2) !\left(1-x^{2}\right)^{r+k-1}} f^{(r-k-1)}(x) .
$$

Proof. The proof is done by induction. It is trivial to see that the identity holds for $r=2$. Suppose that the identity holds for some $r$. Differentiating both sides by $x$, we obtain

$$
\begin{aligned}
r f & (x)^{r-1} f^{\prime}(x) \\
= & \frac{x^{2 r-2} f^{(r)}(x)}{(r-1) !\left(1-x^{2}\right)^{r-1}}+\frac{(2 r-2) x^{2 r-3} f^{(r-1)}(x)}{(r-1) !\left(1-x^{2}\right)^{r}} \\
& +\sum_{k=1}^{r-2} \frac{\sum_{j=0}^{k-1}\left(\begin{array}{c}
k \\
j
\end{array}\right)\left(\begin{array}{c}
r-2 \\
k-j-1
\end{array}\right) x^{2 r-k-2+2 j}}{k(r-k-2) !\left(1-x^{2}\right)^{r+k-1}} f^{(r-k)}(x) \\
& +\sum_{k=1}^{r-2} \frac{\sum_{j=0}^{k-1}(2 r-k-2+2 j)\left(\begin{array}{c}
k \\
j
\end{array}\right)\left(\begin{array}{c}
r-2 \\
k-j-1
\end{array}\right) x^{2 r-k-3+2 j}}{k(r-k-2) !\left(1-x^{2}\right)^{r+k}} f^{(r-k-1)}(x) \\
& +\sum_{k=1}^{r-2} \frac{\sum_{j=0}^{k-1}(3 k-2 j)\left(\begin{array}{c}
k \\
j
\end{array}\right)\left(\begin{array}{c}
r-2 \\
k-j-1
\end{array}\right) x^{2 r-k-1+2 j}}{k(r-k-2) !\left(1-x^{2}\right)^{r+k}} f^{(r-k-1)}(x) \\
= & \frac{x^{2 r-2} f^{(r)}(x)}{(r-1) !\left(1-x^{2}\right)^{r-1}+\frac{2 x^{2 r-3} f^{(r-1)}(x)}{(r-2) !\left(1-x^{2}\right)^{r}}} \\
& +\sum_{k=1}^{r-2} \frac{\sum_{j=0}^{k-1}\left(\begin{array}{c}
k \\
j
\end{array}\right)\left(\begin{array}{c}
r-2 \\
k-j-1
\end{array}\right) x^{2 r-k-2+2 j}}{k(r-k-2) !\left(1-x^{2}\right)^{r+k-1} f^{(r-k)}(x)} \\
& +\sum_{k=2}^{r-1} \frac{\sum_{j=0}^{k-2}(2 r-k-1+2 j)\left(\begin{array}{c}
k-1 \\
j
\end{array}\right)\left(\begin{array}{c}
r-2 \\
k-j-2
\end{array}\right) x^{2 r-k-2+2 j}}{(k-1)(r-k-1) !\left(1-x^{2}\right)^{r+k-1}} f^{(r-k)}(x) \\
& +\sum_{k=2}^{r-1} \frac{\sum_{j=1}^{k-1}(3 k-2 j-1)\left(\begin{array}{c}
k-1 \\
j-1
\end{array}\right)\left(\begin{array}{c}
r-2 \\
k-j-1
\end{array}\right) x^{2 r-k-2+2 j}}{(k-1)(r-k-1) !\left(1-x^{2}\right)^{r+k-1}} f^{(r-k)}(x)
\end{aligned}
$$




$$
=\frac{x^{2 r-2} f^{(r)}(x)}{(r-1) !\left(1-x^{2}\right)^{r-1}}+r \sum_{k=1}^{r-1} \frac{\sum_{j=0}^{k-1}\left(\begin{array}{c}
k \\
j
\end{array}\right)\left(\begin{array}{c}
r-1 \\
k-j-1
\end{array}\right) x^{2 r-k-2+2 j}}{k(r-k-1) !\left(1-x^{2}\right)^{r+k-1}} f^{(r-k)}(x) .
$$

Together with (3), we get

$$
\begin{aligned}
f(x)^{r+1} & =\frac{x^{2}}{1-x^{2}} f(x)^{r-1} f^{\prime}(x) \\
& =\frac{x^{2}}{1-x^{2}}\left(\frac{x^{2 r-2} f^{(r)}(x)}{r !\left(1-x^{2}\right)^{r-1}}+\sum_{k=1}^{r-1} \frac{\sum_{j=0}^{k-1}\left(\begin{array}{c}
k \\
j
\end{array}\right)\left(\begin{array}{c}
r-1 \\
k-j-1
\end{array}\right) x^{2 r-k-2+2 j}}{k(r-k-1) !\left(1-x^{2}\right)^{r+k-1}} f^{(r-k)}(x)\right) \\
& =\frac{x^{2 r} f^{(r)}(x)}{r !\left(1-x^{2}\right)^{r}}+\sum_{k=1}^{r-1} \frac{\sum_{j=0}^{k-1}\left(\begin{array}{c}
k \\
j
\end{array}\right)\left(\begin{array}{c}
r-1 \\
k-j-1
\end{array}\right) x^{2 r-k+2 j}}{k(r-k-1) !\left(1-x^{2}\right)^{r+k}} f^{(r-k)}(x) .
\end{aligned}
$$

In general, we can state the following.

Theorem 3. Let $r \geq 2$. Then for $n \geq 3 r-5$, we have

$$
\begin{aligned}
& \sum_{l=0}^{2 r-3}(-1)^{l}\left(\begin{array}{c}
2 r-3 \\
l
\end{array}\right) \sum_{\substack{j_{1}+\cdots+j_{r}=n-2 l \\
j_{1}, \ldots, j_{r} \geq 1}} B_{j_{1}} \cdots B_{j_{r}} \\
& \quad=\sum_{k=1}^{r-1}(-1)^{k-1} \frac{n-2 k-r+3}{r-1}\left(\begin{array}{c}
n-2 k+1 \\
r-k-1
\end{array}\right)\left(\begin{array}{c}
n-k-2 r+3 \\
k-1
\end{array}\right) B_{n-2 k-r+3} .
\end{aligned}
$$

Proof. By Lemma 1 we get

$$
\begin{aligned}
\left(1-x^{2}\right)^{2 r-3} f(x)^{r}=(1- & \left.x^{2}\right)^{r-2} \frac{x^{2 r-2} f^{(r-1)}(x)}{(r-1) !} \\
& +\sum_{k=1}^{r-2}\left(1-x^{2}\right)^{r-k-2} \frac{\sum_{j=0}^{k-1}\left(\begin{array}{c}
k \\
j
\end{array}\right)\left(\begin{array}{c}
r-2 \\
k-j-1
\end{array}\right) x^{2 r-k-2+2 j}}{k(r-k-2) !} f^{(r-k-1)}(x) .
\end{aligned}
$$

Since $B_{0}=0$, the left-hand side of (9) is equal to

$$
\left(1-x^{2}\right)^{2 r-3} \sum_{n=0}^{\infty} \sum_{\substack{j_{1}+\cdots+j_{r}=n \\
j_{1}, \ldots, j_{r} \geq 0}} B_{j_{1}} \cdots B_{j_{r}} x^{n}=\sum_{l=0}^{2 r-3} \sum_{n=2 l}^{\infty}(-1)^{l}\left(\begin{array}{c}
2 r-3 \\
l
\end{array}\right) \sum_{\substack{j_{1}+\ldots+j_{r}=n-2 l \\
j_{1}, \ldots, j_{r} \geq 1}} B_{j_{1}} \cdots B_{j_{r}} x^{n} .
$$

On the other hand,

$$
\begin{aligned}
& \left(1-x^{2}\right)^{r-2} \frac{x^{2 r-2} f^{(r-1)}(x)}{(r-1) !} \\
& =\sum_{i=0}^{r-2}\left(\begin{array}{c}
r-2 \\
i
\end{array}\right) x^{2 i} \frac{x^{2 r-2}}{(r-1) !} \sum_{n=r-1}^{\infty} \frac{n !}{(n-r+1) !} B_{n} x^{n-r+1} \\
& =\frac{1}{(r-1) !} \sum_{i=0}^{r-2}\left(\begin{array}{c}
r-2 \\
i
\end{array}\right) \sum_{n=2 r+2 i-2}^{\infty} \frac{(n-r-2 i+1) !}{(n-2 r-2 i+2) !} B_{n-r-2 i+1} x^{n} .
\end{aligned}
$$


For $i=r-2$, we have

$$
\begin{aligned}
& \frac{(-1)^{r-2}}{(r-1) !} \sum_{n=4 r-6}^{\infty} \frac{(n-3 r+5) !}{(n-4 r+6) !} B_{n-3 r+5} x^{n} \\
& =(-1)^{r-2} \sum_{n=3 r-5}^{\infty} \frac{n-3 r+5}{r-1}\left(\begin{array}{c}
n-3 r+4 \\
r-2
\end{array}\right) B_{n-3 r+5} x^{n},
\end{aligned}
$$

which yields the term for $k=r-1$ on the right-hand side of the identity in Theorem 3 . Notice that

$$
\left(\begin{array}{l}
\gamma^{\prime} \\
\gamma
\end{array}\right)=0 \quad\left(\gamma^{\prime}<\gamma\right) .
$$

The second term of the right-hand side of (9) is

$$
\begin{aligned}
& \sum_{k=1}^{r-2} \sum_{i=0}^{r-k-2}(-1)^{i}\left(\begin{array}{c}
r-k-2 \\
i
\end{array}\right) x^{2 i} \frac{1}{k(r-k-2) !} \sum_{j=0}^{k-1}\left(\begin{array}{l}
k \\
j
\end{array}\right)\left(\begin{array}{c}
r-2 \\
k-j-1
\end{array}\right) x^{2 r-k-2+2 j} \\
& \quad \times \sum_{n=r-k-1}^{\infty} \frac{n !}{(n-r+k+1) !} B_{n} x^{n-r+k+1} \\
& =\sum_{i=0}^{r-3} \sum_{j=0}^{r-i-3} \sum_{k=j}^{r-i-3} \frac{(-1)^{i}}{(k+1)(r-k-3) !(n-2 r+k-2 i-2 j+3) !}\left(\begin{array}{c}
r-k-3 \\
i
\end{array}\right) \\
& \quad \times\left(\begin{array}{c}
k+1 \\
j
\end{array}\right)\left(\begin{array}{c}
r-2 \\
k-j
\end{array}\right) \sum_{n=2 r+2 i+2 j-k-3}(n-r-2 i-2 j+1) ! B_{n-r-2 i-2 j+1} x^{n} \\
& =\sum_{i=0}^{r-3} \sum_{\kappa=i+1}^{r-2} \sum_{k=\kappa-i-1}^{r-i-3} \frac{(-1)^{i}}{(k+1)(r-k-3) !(n-2 r+k-2 \kappa+5) !}\left(\begin{array}{c}
r-k-3 \\
i
\end{array}\right) \\
& \quad \times\left(\begin{array}{c}
k+1 \\
k-i-1
\end{array}\right)\left(\begin{array}{c}
r-2 \\
k-\kappa+i+1
\end{array}\right) \sum_{n=2 r+2 \kappa-k-5}(n-r-2 \kappa+3) ! B_{n-r-2 \kappa+3} x^{n} .
\end{aligned}
$$

Together with the first term of the right-hand side of (9) we can prove that

$$
\begin{aligned}
& \frac{(-1)^{k-1}}{(r-1) !}\left(\begin{array}{c}
r-2 \\
k-1
\end{array}\right) \frac{(n-r-2 k+3) !}{(n-2 r-2 k+4) !} \\
& \quad+\sum_{i=0}^{k-1} \sum_{l=k-i-1}^{r-i-3} \frac{(-1)^{k-1}}{(l+1)(r-l-3) !(n-2 r+l-2 k+5) !} \\
& \quad \times\left(\begin{array}{c}
r-l-3 \\
i
\end{array}\right)\left(\begin{array}{c}
l+1 \\
k-i-1
\end{array}\right)\left(\begin{array}{c}
r-2 \\
l-k+i+1
\end{array}\right)(n-r-2 k+3) ! \\
& =(-1)^{k-1} \frac{n-2 k-r+3}{r-1}\left(\begin{array}{c}
n-2 k+1 \\
r-k-1
\end{array}\right)\left(\begin{array}{c}
n-k-2 r+3 \\
k-1
\end{array}\right) .
\end{aligned}
$$

Then the proof is done. 


\section{Another result}

In this section, we shall give an expression of $\sum_{\substack{j_{1}+\cdots+j_{r}=n \\ j_{1}, \ldots, j_{r} \geq 1}} B_{j_{1}} \cdots B_{j_{r}}$.

The left-hand side of (3) is equal to

$$
\left(\sum_{u=0}^{\infty} B_{u} x^{u}\right)\left(\sum_{v=0}^{\infty} B_{v} x^{v}\right)=\sum_{n=0}^{\infty} \sum_{j=0}^{n} B_{j} B_{n-j} x^{n} .
$$

The right-hand side of (3) is equal to

$$
\begin{aligned}
& x^{2}\left(\sum_{j=0}^{\infty} x^{2 j}\right)\left(\sum_{m=1}^{\infty} m B_{m} x^{m-1}\right) \\
& =x \sum_{n=0}^{\infty}\left(\sum_{m=0}^{n} \frac{1+(-1)^{m}}{2}(n-m) B_{n-m}\right) x^{n} \\
& =\sum_{n=1}^{\infty}\left(\sum_{m=0}^{\left\lfloor\frac{n-1}{2}\right\rfloor}(n-2 m-1) B_{n-2 m-1}\right) x^{n} .
\end{aligned}
$$

Comparing the coefficients of both sides, we have the following.

Theorem 4. For $n \geq 2$, we have

$$
\sum_{j=1}^{n-1} B_{j} B_{n-j}=\sum_{m=0}^{\left\lfloor\frac{n-1}{2}\right\rfloor}(n-2 m-1) B_{n-2 m-1}
$$

In general, we have the following.

Theorem 5. For $n \geq r \geq 2$, we have

$$
\sum_{\substack{j_{1}+\cdots, j_{r}=n \\
j_{1}, \ldots, j_{r} \geq 1}} B_{j_{1}} \cdots B_{j_{r}}=\sum_{m=0}^{\left\lfloor\frac{n-r+1}{2}\right\rfloor}\left(\begin{array}{c}
n-m-1 \\
r-2
\end{array}\right)\left(\begin{array}{c}
m+r-2 \\
r-2
\end{array}\right) \frac{n-2 m-r+1}{r-1} B_{n-2 m-r+1} .
$$

Proof. The left-hand side of (8) in Lemma 1 is equal to

$$
\sum_{n=0}^{\infty} \sum_{\substack{j_{1}+\cdots+j_{r}=n \\ j_{1}, \ldots, j_{r} \geq 1}} B_{j_{1}} \cdots B_{j_{r}} x^{n}
$$

The first term on the right-hand side of (8) in Lemma 1 is equal to

$$
\begin{aligned}
& \frac{x^{2 r-2} f^{(r-1)}(x)}{(r-1) !\left(1-x^{2}\right)^{r-1}} \\
& =\frac{x^{2 r-2}}{(r-1) !} \sum_{i=0}^{\infty}\left(\begin{array}{c}
i+r-2 \\
r-2
\end{array}\right) x^{2 i} \sum_{m=0}^{\infty} \frac{(m+r-1) !}{m !} B_{m+r-1} x^{m}
\end{aligned}
$$




$$
\begin{aligned}
= & \frac{x^{2 r-2}}{(r-1) !} \sum_{k=0}^{\infty} \frac{1}{(r-2) ! 2^{r-2}} \frac{(k+2 r-4) ! !}{k ! !} \frac{1+(-1)^{k}}{2} x^{k} \\
& \times \sum_{m=0}^{\infty} \frac{(m+r-1) !}{m !} B_{m+r-1} x^{m} \\
= & \frac{1}{(r-1) !(r-2) ! 2^{r-2}} \sum_{n=2 r-2}^{\infty} \sum_{m=r-1}^{n-r+1} \frac{(n-m+r-3) ! !}{(n-m-r+1) ! !} \\
& \quad \times \frac{1+(-1)^{n-m-r+1}}{2} \frac{m !}{(m-r+1) !} B_{m} x^{n} .
\end{aligned}
$$

Concerning the second term, we have

$$
\begin{aligned}
& \frac{\sum_{j=0}^{k-1}\left(\begin{array}{l}
k \\
j
\end{array}\right)\left(\begin{array}{c}
r-2 \\
k-j-1
\end{array}\right) x^{2 r-k-2+2 j}}{\left(1-x^{2}\right)^{r+k-1}} f^{(r-k-1)}(x) \\
&=\sum_{j=0}^{k-1}\left(\begin{array}{c}
k \\
j
\end{array}\right)\left(\begin{array}{c}
r-2 \\
k-j-1
\end{array}\right) x^{2 r-k-2+2 j} \sum_{i=0}^{\infty}\left(\begin{array}{c}
i+r+k-2 \\
r+k-2
\end{array}\right) x^{2 i} \\
& \quad \times \sum_{m=0}^{\infty} \frac{(m+r-k-1) !}{m !} B_{m+r-k-1} x^{m} \\
&=\frac{1}{(r+k-2) ! 2^{r+k-2}} \sum_{j=0}^{k-1}\left(\begin{array}{c}
k \\
j
\end{array}\right)\left(\begin{array}{c}
r-2 \\
k-j-1
\end{array}\right) \sum_{n=2 r-k-2+2 j}^{\infty} \sum_{m=0}^{n-2 r+k+2-2 j} \\
& \frac{(n-m+3 k-2-2 j) ! !}{(n-m-2 r+k+2-2 j) ! !} \frac{1+(-1)^{n-m+k}}{2} \frac{(m+r-k-1) !}{m !} B_{m+r-k-1} x^{n} .
\end{aligned}
$$

Since

$$
\frac{(n-m+r+2 k-3-2 j) ! !}{(n-m-r+k+3-2 j) ! !}=0
$$

if $m=n-2 r+k+2-2 j(j=1,2, \ldots, k-2)$, this is equal to

$$
\begin{aligned}
& \frac{1}{(r+k-2) ! 2^{r+k-2}} \sum_{j=0}^{k-1}\left(\begin{array}{c}
k \\
j
\end{array}\right)\left(\begin{array}{c}
r-2 \\
k-j-1
\end{array}\right) \sum_{n=2 r-k-2}^{\infty} \sum_{m=0}^{n-2 r+k+2} \\
& \times \frac{(n-m+3 k-2-2 j) ! !}{(n-m+k) ! !} \frac{1+(-1)^{n-m+k}}{2} \frac{(m+r-k-1) !}{m !} B_{m+r-k-1} x^{n} \\
& =\frac{1}{(r+k-2) ! 2^{r+k-2}} \sum_{n=2 r-k-2}^{\infty} \sum_{m=r-k-1}^{n-r+1} \frac{(n-m+r-1) ! !}{(n-m-r+1) ! !}\left(\begin{array}{c}
r+k-2 \\
k-1
\end{array}\right) \\
& \quad \times \frac{(n-m+r-3) ! !}{(n-m+r-2 k-1) ! !} \frac{1+(-1)^{n-m-r+1}}{2} \frac{m !}{(m-r+k+1) !} B_{m} x^{n} .
\end{aligned}
$$

Therefore, the right-hand side of the relation in Theorem 4 is

$$
\begin{aligned}
& \frac{1}{(r-1) !(r-2) ! 2^{r-2}} \sum_{n=2 r-2}^{\infty} \sum_{m=r-1}^{n-r+1} \frac{(n-m+r-3) ! !}{(n-m-r+1) ! !} \frac{1+(-1)^{n-m-r+1}}{2} \\
& \times \frac{m !}{(m-r+1) !} B_{m} x^{n}
\end{aligned}
$$




$$
\begin{aligned}
+ & \sum_{k=1}^{r-1} \frac{1}{k(r-k-2) !} \frac{1}{(r+k-2) ! 2^{r+k-2}} \sum_{n=2 r-k-2}^{\infty} \\
& \sum_{m=r-k-1}^{n-r+1} \frac{(n-m+r-1) ! !}{(n-m-r+1) ! !}\left(\begin{array}{c}
r+k-2 \\
k-1
\end{array}\right) \frac{(n-m+r-3) ! !}{(n-m+r-2 k-1) ! !} \\
& \times \frac{1+(-1)^{n-m-r+1}}{2} \frac{m !}{(m-r+k+1) !} B_{m} x^{n} \\
& \frac{1}{(r-1) !(r-2) ! 2^{r-2}} \sum_{n=2 r-2}^{\infty} \sum_{m=r-1}^{n-r+1} \frac{(n-m+r-3) ! !}{(n-m-r+1) ! !} \frac{1+1)^{n-m-r+1}}{2} \\
& \times \frac{m !}{(m-r+1) !} B_{m} x^{n} \\
& \sum_{n=r-1}^{\infty} \frac{1}{(r-1) ! 2^{r-2}} \sum_{m=1}^{r-2} \sum_{k=r-m-1}^{r-2} \frac{1}{k !(r-k-2) ! 2^{k}} \frac{(n-m+r-1) ! !}{(n-m-r+1) ! !} \\
& \times \frac{(n-m+r-3) ! !}{(n-m+r-2 k-1) ! !} \frac{1+(-1)^{n-m-r+1}}{2} \frac{m !}{(m-r+k+1) !} B_{m} x^{n} \\
+ & \sum_{n=r-1}^{\infty} \frac{1}{(r-1) ! 2^{r-2}} \sum_{m=r-1}^{n-r+1} \sum_{k=1}^{r-2} \frac{1}{k !(r-k-2) ! 2^{k}} \frac{(n-m+r-1) ! !}{(n-m-r+1) ! !} \\
& \times \frac{(n-m+r-3) ! !}{(n-m+r-2 k-1) ! !} \frac{1+(-1)^{n-m-r+1}}{2} \frac{m !}{(m-r+k+1) !} B_{m} x^{n} .
\end{aligned}
$$

Since for $1 \leq m \leq r-2$ we have

$$
\begin{aligned}
& \frac{1}{(r-1) ! 2^{r-2}} \sum_{k=r-m-1}^{r-2} \frac{1}{k !(r-k-2) ! 2^{k}} \frac{(n-m+r-1) ! !}{(n-m-r+1) ! !} \\
& \quad \times \frac{(n-m+r-3) ! !}{(n-m+r-2 k-1) ! !} \frac{m !}{(m-r+k+1) !} \\
& =\frac{1}{(r-1) !(r-2) ! 2^{2 r-4}} \frac{(n+m+r-3) ! !}{(n+m-r+1) ! !} \frac{(n-m+r-3) ! !}{(n-m-r+1) ! !} m
\end{aligned}
$$

and for $r-1 \leq m \leq n-r+1$ we have

$$
\begin{aligned}
\frac{1}{(r-1) !(r-2) ! 2^{r-2}} \frac{(n-m+r-3) ! !}{(n-m-r+1) ! !} \frac{m !}{(m-r+1) !} \\
+\frac{1}{(r-1) ! 2^{r-2}} \sum_{k=r-m-1}^{r-2} \frac{1}{k !(r-k-2) ! 2^{k}} \frac{(n-m+r-1) ! !}{(n-m-r+1) ! !} \\
\quad \times \frac{(n-m+r-3) ! !}{(n-m+r-2 k-1) ! !} \frac{m !}{(m-r+k+1) !} \\
=\frac{1}{(r-1) !(r-2) ! 2^{2 r-4}} \frac{(n+m+r-3) ! !}{(n+m-r+1) ! !} \frac{(n-m+r-3) ! !}{(n-m-r+1) ! !} m,
\end{aligned}
$$

By comparing the coefficients, we have

$$
\sum_{\substack{j_{1}+\cdots+j_{r}=n \\ j_{1}, \ldots, j_{r} \geq 1}} B_{j_{1}} \cdots B_{j_{r}}
$$




$$
\begin{aligned}
= & \frac{1}{(r-1) !(r-2) ! 2^{2 r-4}} \\
& \times \sum_{m=0}^{n-r+1} \frac{(n+m+r-3) ! !(n-m+r-3) ! !}{(n+m-r+1) ! !(n-m-r+1) ! !} \frac{1+(-1)^{n-m-r+1}}{2} m F_{m} \\
= & \frac{1}{(r-1) !(r-2) ! 2^{2 r-4}} \\
& \times \sum_{m=0}^{\left.\frac{n-r+1}{2}\right\rfloor} \frac{(2 n-2 m-2) ! !(2 m+2 r-4) ! !}{(2 n-2 m-2 r+2) ! !(2 m) ! !}(n-2 m-r+1) B_{n-2 m-r+1} \\
= & \sum_{m=0}^{\left.\frac{n-r+1}{2}\right\rfloor}\left(\begin{array}{c}
n-m-1 \\
r-2
\end{array}\right)\left(\begin{array}{c}
m+r-2 \\
r-2
\end{array}\right) \frac{n-2 m-r+1}{r-1} B_{n-2 m-r+1} .
\end{aligned}
$$

\section{Some other generating functions}

Another kinds of the generating functions of balancing numbers and Lucas-balancing numbers are given by

$$
b(t):=\frac{e^{\alpha t}-e^{\beta t}}{4 \sqrt{2}}=\sum_{n=0}^{\infty} B_{n} \frac{t^{n}}{n !} \quad \text { and } \quad c(t):=\frac{e^{\alpha t}+e^{\beta t}}{2}=\sum_{n=0}^{\infty} C_{n} \frac{t^{n}}{n !}
$$

because they satisfy the differential equation $y^{\prime \prime}-6 y^{\prime}+y=0$.

Since $b^{\prime}(t)=3 b(t)+c(t)$ and $c^{\prime}(t)=8 b(t)+3 c(t)$, we have for $n \geq 0, B_{n+1}=3 B_{n}+C_{n}$ and $C_{n+1}=8 B_{n}+3 C_{n}$. Since

$$
c(t)^{2}=\frac{e^{2 \alpha t}+e^{2 \beta t}}{4}+\frac{e^{6 t}}{2}
$$

we have

$$
\sum_{n=0}^{\infty} \sum_{k=0}^{n}\left(\begin{array}{l}
n \\
k
\end{array}\right) C_{k} C_{n-k}=\frac{1}{2} \sum_{n=0}^{\infty} C_{n} \frac{(2 t)^{n}}{n !}+\frac{1}{2} \sum_{n=0}^{\infty} \frac{(6 t)^{n}}{n !}
$$

yielding

$$
\sum_{k=0}^{n}\left(\begin{array}{l}
n \\
k
\end{array}\right) C_{k} C_{n-k}=\frac{2^{n} C_{n}+6^{n}}{2} \quad(n \geq 0) .
$$

Similarly, by

$$
b(t)^{2}=\frac{e^{2 \alpha t}+e^{2 \beta t}}{32}-\frac{e^{6 t}}{16}
$$

we have

$$
\sum_{k=0}^{n}\left(\begin{array}{l}
n \\
k
\end{array}\right) B_{k} B_{n-k}=\frac{2^{n} C_{n}-6^{n}}{16} \quad(n \geq 0) .
$$

Since by $2 \alpha+\beta=\alpha+6$ and $\alpha+2 \beta=\beta+6$

$$
b(t)^{3}=\frac{e^{3 \alpha t}-e^{3 \beta t}}{(4 \sqrt{2})^{3}}-\frac{3\left(e^{(2 \alpha+\beta) t}-e^{(\alpha+2 \beta) t}\right)}{(4 \sqrt{2})^{3}}
$$




$$
=\frac{1}{32} \sum_{n=0}^{\infty} 3^{n} B_{n} \frac{t^{n}}{n !}-\frac{3}{32} \sum_{n=0}^{\infty} \sum_{k=0}^{n}\left(\begin{array}{l}
n \\
k
\end{array}\right) 6^{n-k} B_{k} \frac{t^{n}}{n !}
$$

we have

$$
\sum_{\substack{k_{1}+k_{2}+k_{3}=n \\
k_{1}, k_{2}, k_{3} \geq 1}}\left(\begin{array}{c}
n \\
k_{1}, k_{2}, k_{3}
\end{array}\right) B_{k_{1}} B_{k_{2}} B_{k_{3}}=\frac{1}{32}\left(3^{n} B_{n}-3 \sum_{k=0}^{n}\left(\begin{array}{l}
n \\
k
\end{array}\right) 6^{n-k} B_{k}\right) .
$$

Notice that $B_{0}=0$. Similarly, we can obtain that

$$
\sum_{\substack{k_{1}+k_{2}+k_{3}=n \\
k_{1}, k_{2}, k_{3} \geq 0}}\left(\begin{array}{c}
n \\
k_{1}, k_{2}, k_{3}
\end{array}\right) C_{k_{1}} C_{k_{2}} C_{k_{3}}=\frac{1}{4}\left(3^{n} C_{n}+3 \sum_{k=0}^{n}\left(\begin{array}{l}
n \\
k
\end{array}\right) 6^{n-k} C_{k}\right) .
$$

Let $r \geq 1$. If $r$ is odd, then

$$
\begin{aligned}
b(t)^{r} & =\left(\frac{e^{\alpha t}-e^{\beta t}}{4 \sqrt{2}}\right)^{r} \\
& =\frac{1}{(4 \sqrt{2})^{r}} \sum_{j=0}^{\frac{r-1}{2}}(-1)^{j}\left(\begin{array}{l}
r \\
j
\end{array}\right)\left(e^{((r-j) \alpha+j \beta) t}-e^{(j \alpha+(r-j) \beta) t}\right) \\
& =\frac{1}{(4 \sqrt{2})^{r-1}} \sum_{j=0}^{\frac{r-1}{2}}(-1)^{j}\left(\begin{array}{l}
r \\
j
\end{array}\right) \sum_{n=0}^{\infty} \sum_{k=0}^{n}\left(\begin{array}{l}
n \\
k
\end{array}\right)(6 j)^{n-k}(r-2 j)^{k} B_{k} \frac{t^{n}}{n !} .
\end{aligned}
$$

Therefore, we get

$$
\sum_{\substack{k_{1}+\cdots+k_{r}=n \\
k_{1}, \ldots, k_{r} \geq 1}}\left(\begin{array}{c}
n \\
k_{1}, \ldots, k_{r}
\end{array}\right) B_{k_{1}} \cdots B_{k_{r}}=\frac{1}{(4 \sqrt{2})^{r-1}} \sum_{j=0}^{\frac{r-1}{2}}(-1)^{j}\left(\begin{array}{l}
r \\
j
\end{array}\right) \sum_{k=0}^{n}\left(\begin{array}{l}
n \\
k
\end{array}\right)(6 j)^{n-k}(r-2 j)^{k} B_{k} .
$$

Similarly, we get

$$
\sum_{\substack{k_{1}+\cdots+k_{r}=n \\
k_{1}, \ldots, k_{r} \geq 0}}\left(\begin{array}{c}
n \\
k_{1}, \ldots, k_{r}
\end{array}\right) C_{k_{1}} \cdots C_{k_{r}}=\frac{1}{2^{r-1}} \sum_{j=0}^{\frac{r-1}{2}}\left(\begin{array}{l}
r \\
j
\end{array}\right) \sum_{k=0}^{n}\left(\begin{array}{l}
n \\
k
\end{array}\right)(6 j)^{n-k}(r-2 j)^{k} C_{k}
$$

If $r$ is even, then

$$
\begin{aligned}
b(t)^{r}= & \frac{1}{(4 \sqrt{2})^{r}}\left(\sum_{j=0}^{\frac{r}{2}-1}(-1)^{j}\left(\begin{array}{l}
r \\
j
\end{array}\right)\left(e^{((r-j) \alpha+j \beta) t}+e^{(j \alpha+(r-j) \beta) t}\right)\right. \\
& \left.+(-1)^{\frac{r}{2}}\left(\begin{array}{c}
r \\
\frac{r}{2}
\end{array}\right) e^{\left(\frac{r}{2} \alpha+\frac{r}{2} \beta\right) t}\right) \\
= & \frac{1}{(4 \sqrt{2})^{r}}\left(2 \sum_{j=0}^{\frac{r}{2}-1}(-1)^{j}\left(\begin{array}{l}
r \\
j
\end{array}\right) \sum_{n=0}^{\infty} \sum_{k=0}^{n}\left(\begin{array}{l}
n \\
k
\end{array}\right)(6 j)^{n-k}(r-2 j)^{k} C_{k} \frac{t^{n}}{n !}\right. \\
& \left.+(-1)^{\frac{r}{2}}\left(\begin{array}{c}
r \\
\frac{r}{2}
\end{array}\right) \sum_{n=0}^{\infty}(3 r)^{n} \frac{t^{n}}{n !}\right) .
\end{aligned}
$$


Therefore, we get

$$
\begin{aligned}
\sum_{\substack{k_{1}+\cdots+k_{r}=n \\
k_{1}, \ldots, k_{r} \geq 1}}\left(\begin{array}{c}
n \\
k_{1}, \ldots, k_{r}
\end{array}\right) B_{k_{1}} \cdots B_{k_{r}} \\
=\frac{1}{(4 \sqrt{2})^{r}}\left(2 \sum_{j=0}^{\frac{r}{2}-1}(-1)^{j}\left(\begin{array}{l}
r \\
j
\end{array}\right) \sum_{k=0}^{n}\left(\begin{array}{l}
n \\
k
\end{array}\right)(6 j)^{n-k}(r-2 j)^{k} C_{k}+(-1)^{\frac{r}{2}}\left(\begin{array}{c}
r \\
\frac{r}{2}
\end{array}\right)(3 r)^{n}\right) .
\end{aligned}
$$

Similarly, we get

$$
\begin{aligned}
& \sum_{\substack{k_{1}+\cdots+k_{r}=n \\
k_{1}, \ldots, k_{r} \geq 0}}\left(\begin{array}{c}
n \\
k_{1}, \ldots, k_{r}
\end{array}\right) C_{k_{1}} \cdots C_{k_{r}} \\
& =\frac{1}{2^{r}}\left(2 \sum_{j=0}^{\frac{r}{2}-1}\left(\begin{array}{l}
r \\
j
\end{array}\right) \sum_{k=0}^{n}\left(\begin{array}{l}
n \\
k
\end{array}\right)(6 j)^{n-k}(r-2 j)^{k} C_{k}+\left(\begin{array}{c}
r \\
\frac{r}{2}
\end{array}\right)(3 r)^{n}\right) .
\end{aligned}
$$

\section{More general cases}

Let $\left\{u_{n}\right\}_{n \geq 0}$ and $\left\{v_{n}\right\}_{n \geq 0}$ be integer sequences, satisfying the same recurrence relation: $u_{n}=a u_{n-1}+b u_{n-2}(n \geq 2)$ and $v_{n}=a v_{n-1}+b v_{n-2}(n \geq 2)$ with initial values $u_{0}, u_{1}, v_{0}$ and $v_{1}$. If the general terms are given by

$$
u_{n}=\frac{\alpha^{n}-\beta^{n}}{\alpha-\beta} \quad \text { and } \quad v_{n}=\alpha^{n}+\beta^{n} \quad(n \geq 0)
$$

where

$$
\alpha=\frac{a+\sqrt{a^{2}+4 b}}{2} \text { and } \beta=\frac{a-\sqrt{a^{2}+4 b}}{2},
$$

we can set $u_{0}=0, u_{1}=1, v_{0}=2$ and $v_{1}=a$. Then, the generating functions of $u_{n}$ and $v_{n}$ are given by

$$
u(t):=\frac{e^{\alpha t}-e^{\beta t}}{\sqrt{a^{2}+4 b}}=\sum_{n=0}^{\infty} u_{n} \frac{t^{n}}{n !} \quad \text { and } \quad v(t):=e^{\alpha t}+e^{\beta t}=\sum_{n=0}^{\infty} v_{n} \frac{t^{n}}{n !},
$$

respectively, because they satisfy the differential equation $y^{\prime \prime}-a y^{\prime}-b y=0$.

Our main results can be stated as follows. The proof is similar to that in the previous section and is omitted.

Theorem 6. If $r$ is odd, then

$$
\begin{aligned}
& \sum_{\substack{k_{1}+\cdots+k_{r}=n \\
k_{1}, \ldots, k_{r} \geq 1}}\left(\begin{array}{c}
n \\
k_{1}, \ldots, k_{r}
\end{array}\right) u_{k_{1}} \cdots u_{k_{r}} \\
& =\frac{1}{\left(\sqrt{a^{2}+4 b}\right)^{r-1}} \sum_{k=0}^{n}\left(\begin{array}{l}
n \\
k
\end{array}\right) \sum_{j=0}^{\frac{r-1}{2}}(-1)^{j}\left(\begin{array}{l}
r \\
j
\end{array}\right)(a j)^{n-k}(r-2 j)^{k} u_{k}
\end{aligned}
$$

and 


$$
\sum_{\substack{k_{1}+\cdots+k_{r}=n \\
k_{1}, \ldots, k_{r} \geq 0}}\left(\begin{array}{c}
n \\
k_{1}, \ldots, k_{r}
\end{array}\right) v_{k_{1}} \cdots v_{k_{r}}=\sum_{k=0}^{n}\left(\begin{array}{l}
n \\
k
\end{array}\right) \sum_{j=0}^{\frac{r-1}{2}}\left(\begin{array}{l}
r \\
j
\end{array}\right)(a j)^{n-k}(r-2 j)^{k} v_{k} .
$$

If $r$ is even, then

$$
\begin{aligned}
\sum_{\substack{k_{1}+\cdots+k_{r}=n \\
k_{1}, \ldots, k_{r} \geq 1}}\left(\begin{array}{c}
n \\
k_{1}, \ldots, k_{r}
\end{array}\right) u_{k_{1}} \cdots u_{k_{r}} \\
=\frac{1}{\left(\sqrt{a^{2}+4 b}\right)^{r}}\left(\sum_{k=0}^{n}\left(\begin{array}{l}
n \\
k
\end{array}\right) \sum_{j=0}^{\frac{r}{2}-1}(-1)^{j}\left(\begin{array}{c}
r \\
j
\end{array}\right)(a j)^{n-k}(r-2 j)^{k} v_{k}\right. \\
\left.+(-1)^{\frac{r}{2}}\left(\begin{array}{c}
r \\
\frac{r}{2}
\end{array}\right)\left(\frac{a r}{2}\right)^{n}\right)
\end{aligned}
$$

and

$$
\sum_{\substack{k_{1}+\cdots+k_{r}=n \\
k_{1}, \ldots, k_{r} \geq 0}}\left(\begin{array}{c}
n \\
k_{1}, \ldots, k_{r}
\end{array}\right) v_{k_{1}} \cdots v_{k_{r}}=\sum_{k=0}^{n}\left(\begin{array}{l}
n \\
k
\end{array}\right) \sum_{j=0}^{\frac{r}{2}-1}\left(\begin{array}{l}
r \\
j
\end{array}\right)(a j)^{n-k}(r-2 j)^{k} v_{k}+\left(\begin{array}{c}
r \\
\frac{r}{2}
\end{array}\right)\left(\frac{a r}{2}\right)^{n}
$$

\section{References}

[1] Agoh, T. \& Dilcher, K. (2007). Convolution identities and lacunary recurrences for Bernoulli numbers, J. Number Theory, 124, 105-122.

[2] Agoh, T. \& Dilcher, K. (2009). Higher-order recurrences for Bernoulli numbers, J. Number Theory, 129, 1837-1847.

[3] Agoh, T. \& Dilcher, K. (2014). Higher-order convolutions for Bernoulli and Euler polynomials, J. Math. Anal. Appl., 419, 1235-1247.

[4] Behera, A. \& Panda, G. K. (1999). On the square roots of triangular numbers, Fibonacci Quart., 37, 98-105.

[5] Finkelstein, R. (1965). The house problem, Amer. Math. Monthly, 72, 1082-1088.

[6] Komatsu, T. (2015). Higher-order convolution identities for Cauchy numbers of the second kind, Proc. Jangjeon Math. Soc., 18, 369-383.

[7] Komatsu, T. (2016). Higher-order convolution identities for Cauchy numbers, Tokyo J. Math., 39, 15 pages.

[8] Komatsu, T., Masakova, Z. \& Pelantova, E. (2014). Higher-order identities for Fibonacci numbers, Fibonacci Quart., 52 (5), 150-163. 
[9] Komatsu, T. \& Szalay, L. (2014). Balancing with binomial coefficients, Intern. J. Number Theory, 10, 1729-1742.

[10] Komatsu, T. \& Simsek, Y. (2016). Third and higher order convolution identities for Cauchy numbers, Filomat, 30, 1053-1060.

[11] Liptai, K. (2004). Fibonacci Balancing numbers, Fibonacci Quart., 42, 330-340.

[12] Liptai, K., Luca, F., Pintér, Á. \& Szalay, L. (2009). Generalized balancing numbers, Indag. Math. (N.S.), 20, 87-100.

[13] Panda, G. K. (2009). Some fascinating properties of balancing numbers, In: Proc. of Eleventh Internat. Conference on Fibonacci Numbers and Their Applications, Cong. Numerantium, 194, 185-189.

[14] Patel, B. K. \& Ray, P. K. (2016). The period, rank and order of the sequence of balancing numbers modulo m, Math. Rep. (Bucur.), 18 (3), Article No.9. 